Meta

Journal des tradlucteurs

Translators' Journal

\title{
Terminologie de la télédétection aérospatiale
}

\section{Serge Paul}

Volume 30, numéro 4, décembre 1985

URI : https://id.erudit.org/iderudit/003801ar

DOI : https://doi.org/10.7202/003801ar

Aller au sommaire du numéro

Éditeur(s)

Les Presses de l'Université de Montréal

ISSN

0026-0452 (imprimé)

1492-1421 (numérique)

Découvrir la revue

Citer cet article

Paul, S. (1985). Terminologie de la télédétection aérospatiale. Meta, 30(4),

364-379. https://doi.org/10.7202/003801ar d'utilisation que vous pouvez consulter en ligne.

https://apropos.erudit.org/fr/usagers/politique-dutilisation/ 


\section{TERMINOLOGIE DE LA TÉLÉDÉTECTION AÉROSPATIALE}

La terminologie de la télédétection aérospatiale fait l'objet d'un traitement très précis depuis la création en 1978, sous l'impulsion du professeur Serge Paul, de la Commission ministérielle du même nom, rattachée au ministère de l'Éducation nationale. Elle regroupe des experts d'organismes et d'administrations qui ont recours à cette technique notamment le ministère de l'Environnement, la Société française de photogrammétrie et de télédétection, l'Agence spatiale européenne, le Centre national d'études spatiales, l'Institut national agronomique de Paris - Grignon, etc. Trois types de documents font référence actuellement en France sur ce sujet :

- le dictionnaire de télédétection aérospatiale, sous la direction du professeur Serge Paul, qui regroupe près de 1200 termes français et leur définition, assortis de leur équivalent en anglo-américain ;

- l'arrêté de terminologie récapitulatif des travaux de la commission ministérielle de terminologie (136 termes) parus le 20 octobre 1984 ;

- la liste des termes français et anglo-américains du satellite SPOT proposés par la commission de terminologie ; ce travail est particulièrement important car il a une portée scientifique et commerciale immédiate, le satellite devant être lancé par la France en octobre. La commission de terminologie et le CNES à l'issue de ce travail terminologique commun publieront ce vocabulaire dans le Guide des usagers de SPOT, et au Journal officiel de la République française : alliance de la précision scientifique et de l'efficacité commerciale. 


\section{LES TRAVAUX DE LA SECONDE COMMISSION DE TERMINOLOGIE DE LA} TÉLÉDETECTION AÉROSPATIALE

Le ministère de l'Éducation nationale et le Secrétariat d'État aux universités ont homologué par l'arrêté du 29 septembre 1984 une liste de termes de vocabulaire de la télédétection aérospatiale retenus au 31 décembre 1983 et qui reprend les arrêtés du 28 novembre 1980 et du 14 mai 1982.

La Commission ministérielle de terminologie a entrepris une $3^{\mathrm{e}}$ tranche de travaux à la suite de la décision du 23 décembre 1982 (publiée au B.O.E.N. no 5 du 3 février 1983) portant nomination des membres de la seconde commission. La présidence de celle-ci a été confiée à M. Serge Paul, professeur à l'Université de Paris VIII.

Les dossiers de termes traités ont fait l'objet de trois rapports en février 1980 (54 pages), novembre 1981 (69 pages) et décembre 1983 (78 pages), de la direction générale des enseignements supérieurs et de la recherche. Au cours de ces travaux, une priorité a été donnée d'une part aux notions fondamentales à partir desquelles les méthodes et techniques de la télédétection peuvent être retenues sans ambiguïté, d'autre part aux événements scientifiques remarquables (navette spatiale, programme télédétection en hyperfréquence de l'Agence spatiale européenne, simulations SPOT, par exemple), générateurs de néologismes français et anglo-américains.

Au total, une liste de 139 termes français est arrêtée, lesquels sont présentés sous forme d'articles définitoires (75) ou introduits à propos d'une note à la définition (64). Par ce traitement terminologique, une centaine de termes anglais sont remplacés; de même pour seize termes français fautifs dont l'emploi est déconseillé pour des raisons précises.

On remarquera un double domaine d'appartenance sémantique de ces termes, à la télédétection, considérée comme grand générique, et à la télédétection électromagnétique, prise comme domaine spécifique.

L'enrichissement de ce vocabulaire est le fruit d'une collaboration associant plusieurs ministères et organismes nationaux dont les activités professionnelles internationales requièrent des équivalences entre concepts anglais et français.

Pour mieux faire apparaître l'incidence de cet apport néologique, rappelons que le domaine de la télédétection aérospatiale était couvert en 1980 par environ 1150 termes ${ }^{1}$.

Le ministère de l'Éducation nationale et le Secrétariat d'État chargé des universités entendent donner la plus large audience à ce vocabulaire de la télédétection aérospatiale dans l'optique d'une politique de la promotion du français langue scientifique et de la diffusion de la culture scientifique et technique.

SERGe PaUl

\section{LISTE 1}

Affichage en couleur, n.m.

Domaine : Télédétection.

Définition : Représentation colorée d'un ensemble de données sur un *visuel.

Note : Le terme *visuel a été choisi de préférence à console de visualisation pour sa brièveté.

Voir aussi : Afficher.

Anglo-américain : Colo(u)r display.

Afficher, v. tr.

Domaine : Télédétection.

Synonyme :Visualiser, v. tr.

Définition : Représenter des données sur un *visuel. 
Note : "Visualiser est défini, par arrêté du 22 décembre 1981 relatif à l'enrichissement du vocabulaire de l'informatique (Journal officiel de la République française du 17 janvier 1982), de la façon suivante : inscrire les résultats d'un traitement sur un *visuel.

Anglo-américain : To display.

Amélioration d'image, n.f.

Domaine : Télédétection électromagnétique.

Définition : Ensemble de traitements destinés à rendre une *image plus appropriée à la finalité de l'analyse.

Note : C'est la finalité de l'analyse qui donne un sens à la notion d'amélioration d'image et, dans une certaine mesure, oriente les procédures opératoire.

Anglo-américain : Image improvement.

Angle d'éclairage, n.m.

Voir : Angle d'irradiation.

Angle de dépression, n.m.

Domaine : Télédétection électromagnétique.

Définition : Angle défini par l'axe principal du faisceau radar et le plan horizontal de la *plate-forme.

Note : Le terme angle de dépression à l'horizon (en anglo-américain : dip angle) appartient au vocabulaire de la topographie.

Voir aussi : Angle d'irradiation, angle d'inclinaison.

Anglo-américain : Angle of depression, depression angle.

Angle de dépression à l'horizon, n.m.

Voir : Angle de dépression.

Angle de visée, n.m.

Voir : Angle d'irradiation.

Angle d'inclinaison, n.m.

Domaine : Télédétection électromagnétique.

Définition : Angle défini par la verticale de la *plate-forme et l'axe principal du *capteur.

Note : Dans le cas du radar, cet axe principal est celui du faisceau radar et le terme anglais d'inclinaison est synonyme du terme *angle d'irradiation.

Voir aussi : Angle de dépression.

Anglo-américain : Angle of tilt, tilt angle.

Angle d'irradiation, n.m.

Domaine : Télédétection électromagnétique

Définition : Angle défini par la verticale de la *plate-forme et l'axe principal du faisceau radar.

Note : 1 . Cet angle est complémentaire de $1 *$ angle de dépression.

2. Les termes : angle d'éclairage et angle de visée, parfois utilisés, sont moins rigoureux.

Voir aussi : Angle d'inclinaison.

Anglo-américain : Illumination angle.

Calibrage, n.m.

Domaine: Télédétection.

Définition : Détermination de l'appartenance d'une mesure à une classe d'intensité comprise entre deux valeurs limites prédéterminées.

Note : Le terme calibration ne doit pas être employé pour désigner le calibrage.

Voir aussi : Étalonnage.

Anglo-américain : Calibration.

Calibration, n.f.

Voir : Calibrage, étalonnage.

Capteur, n.m.

Domaine : Télédétection électromagnétique.

Définition : Instrument qui recueille de l'énergie radiative provenant de la *scène visée et délivre un signal électrique correspondant et mesurable.

Note : 1. Par assimilation, et bien qu'elle ne délivre pas de signal électronique, on considère la chambre photographique comme un capteur. 
2. Le terme capteur est parfois utilisé à tort pour désigner le *détecteur.

3. Capteur passif (en anglo-américain : passive sensor) : lorsque le contexte impose qu'une distinction soit faite avec les *capteurs actifs, l'adjectif passif est ajouté au terme capteur.

Anglo-américain : (Remote) Sensor.

Capteur actif, n.m.

Domaine : Télédétection électromagnétique.

Définition : *Capteur auquel est incorporé ou associé un émetteur qui irradie la *scène dans la bande spectrale du récepteur.

Note : 1. Le radar et le lidar sont des exemples de capteurs actifs.

2. Dans le cas des capteurs de luminescence, la réception a lieu dans une bande spectrale différente de celle de l'émission.

Anglo-américain : Active sensor.

Capteur de luminescence, n.m.

Voir : Capteur actif.

Capteur en peigne, n.m.

Domaine : Télédétection électromagnétique.

Définition : *Capteur dont le *détecteur est constitué par de nombreuses cellules détectrices alignées qui recoivent simultanément l'énergie radiative en provenance de la *scène.

Note : Le balayage selon une direction orthogonale à l'alignement des cellules détectrices peut être réalisé par le déplacement du *vecteur ou, par exemple, dans le cas d'une *plate-forme d'observation géostationnaire, être fourni par un séquenceur incorporé au *capteur.

Anglo-américain : Push broom sensor.

Capteur passif, n.m.

Voir : Capteur.

Carte infographique, n.f.

Domaine : Télédétection.

Définition : Carte obtenue par *infographie.

Note : Termes à ne pas employer : carte informatique ; carte informatisée.

Anglo-américain : Computer map, computerized map.

Carte informatique, n.f.

Voir : Carte infographique

Carte informatisée, n.f.

Voir : Carte infographique.

Cellule détectrice, n.f.

Voir : Détecteur.

Chambre multibande, n.f.

Domaine : Télédétection électromagnétique.

Définition : Chambre photographique qui permet d'enregistrer simultanément 1 "*image d'une *scène dans différentes bandes spectrales.

Anglo-américain : Multiband camera.

Chambre photographique, n.f.

Voir : Capteur.

Composition colorée, n.f.

Domaine : Télédétection.

Définition : Représentation significative obtenue par une ou plusieurs combinaisons de couleurs.

Note : Les équidensités colorées sont un exemple de composition colorée.

Anglo-américain : Colo(u)r composite.

Console de visualisation, $n . f$

Voir : Affichage en couleur. 
Couloir exploré, n,m.

Domaine : Télédétection électromagnétique.

Définition : Bande de terrain dont l'axe est parallèle à la trace du *vecteur et dont la largeur dépend principalement de l'angle de *scannage ou d'ouverture de faisceau du *capteur.

Note : 1. Les écarts au parallélisme à la trace peuvent dépendre de l'attitude du *vecteur.

2. Le terme anglo-américain swath width se traduit par largeur de couloir.

Anglo-américain : (Scan) Swath.

Détecteur, n.m.

Domaine : Télédétection électromagnétique

Définition : Composant sensible du *apteur.

Note : 1. Dans le cas de la photographie, les éléments photosensibles de l'émulsion jouent le rôle du *détecteur.

2. Dans tous les autres cas, le terme cellule détectrice peut être considéré comme synonyme de *détecteur.

Anglo-américain : Detector.

Dézersement en radar, n.m.

Abrév. : Déversement-radar, n.m.

Domaine : Télédétection électromagnétique.

Synonyme : Rabattement-radar.

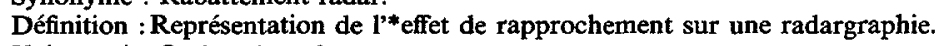

Voir aussi : Ombre de radar.

Anglo-américain : Radar layover.

Déversement-radar, n.m.

Voir : Déversement en radar.

Distance-temps, n.f.

Domaine : Télédétection électromagnétique.

Définition : Distance parcourue par l'onde électromagnétique entre le *capteur actif et la cible, obtenue par la mesure du temps de parcours.

Voir aussi : Distance-temps au sol.

Anglo-américain : Slant range.

Distance-temps au sol, n.f.

Domaine : Télédétection électromagnétique.

Définition : Distance entre la projection de la verticale du *capteur actif et l'intersection du front d'onde, sur la surface de référence au sol.

Note : Ce terme est propre au *radar à visée latérale.

Voir aussi : Distance-temps.

Anglo-américain : Ground range.

Donnée auxiliaire, n.f.

Voir : Réalité de terrain.

Donnée brute, n.f.

Domaine : Télédétection électromagnétique.

Définition : Signal issu d'un "capteur et mis sous une forme, soit analogique, soit numérique, disponible en vue de traitements.

Note : Le terme donnée brute est le plus souvent utilisé au pluriel.

Voir aussi : Donnée corrigée, donnée élaborée, donnée prétraitée, réalité de terrain.

Anglo-américain : Raw data.

Domaine corrigée, n.f.

Domaine : Télédétection électromagnétique.

Définition : *Donnée brute qui a subi un ou plusieurs traitements comportant des corrections radiométriques et/ou géométriques.

Note : 1. Le terme donnée corrigée est le plus souvent utilisé au pluriel.

2. Donnée corrigée est un cas particulier de *donnée prétraitée.

Voir aussi : Donnée élaborée, donnée prétraitée, restauration d'image.

Anglo-américain : Corrected data. 
Donnée de terrain, n.f.

Voir : Réalité de terrain.

Donnée élaborée, n.f.

Domaine : Télédétection électromagnétique.

Définition : État final obtenu par des traitements de *données brutes, de *données prétraitées ou de *données corrigées en vue de leur interprétation.

Note : Le terme donnée élaborée est le plus souvent utilisé an pluriel.

Anglo-américain : Processed data.

Donnée prétraitée, n.f.

Domaine : Télédétection électromagnétique.

Définition : *Donnée brute qui a subi un ou plusieurs traitements optionnels pouvant comporter des corrections en vue de faciliter un ou plusieurs traitements thématiques ultérieurs.

Note : 1. Ces prétraitements consistent, par exemple, à éliminer les effets dus aux varitations d'attitude du * vecteur et/ou aux variations d'éclairement au cours d'une mission.

2. Le terme donnée prétraitée est le plus souvent utilisé au pluriel.

Voir aussi : Donnée corrigée, donnée élaborée, restauration d'image.

Anglo-américain : Pre-processed data.

Effet de mer, n.m.

Domaine : Télédétection électromagnétique.

Définition : Ensemble de phénomènes de rétrodiffusion liés à l'état de surface de la mer, de périodicités différentes, qui se manifeste par un fouillis de luminances sur l'image obtenue.

Note : Un effet semblable peut apparantre également sur les grandes étendues continentales d'eau libre.

Anglo-américain : Sea clutter.

Effet de rapprochement, n.m.

Domaine : Télédétection électromagnétique.

Définition : En technologie radar, délocalisation apparente de certaines parties de la cible écartées du plan horizontal de référence au sol.

Note : Cet effet de rapprochement résulte de l'inclinaison du front d'onde sur ces parties de cible.

Voir aussi : Angle de dépression, déversement en radar.

Anglo-américain : Radar foreshortening.

Épreuve, n.f.

Domaine : Télédétection.

Définition : Copie permanente obtenue sur un support matériel et pouvant être séparée du dispositif de restitution.

Note : Doit être considérée comme épreuve la sortie de table traçante ou celle d'imprimante.

Anglo-américain : Hard copy.

Épreuve minute, n.f.

Domaine : Télédétection électromagnétique.

Définition : *Épreuve rapidement produite de tout ou partie d'une *scène, qui ne restitue pas nécessairement toutes les qualités de l'enregistrement.

Note : Avant de classer l'enregistrement d'une *scène ou d'acquérir les documents correspondants, on examine des épreuves-minutes afin d'apprécier quelques caractéristiques essentielles telles que, par exemple, la localisation et les limites de la *scène, la présence de nuages, des indications sur le contraste et sur le potentiel d'information radiométrique de l'enregistrement.

Voir aussi : Visualisation.

Anglo-américain : Quick look.

Équidensité colorée, n.f.

Voir : Composition colorée.

Etalonnage, n.m.

Domaine : Télédétection.

Définition : Détermination de la relation entre les indications de l'appareil de mesure et les valeurs du mesurande (grandeur à mesurer).

Note : 1. Le terme calibration ne doit pas être employé pour désigner l'étalonnage. 
2. Cet étalonnage peut être effectué par rapport à une source de référence intérieure ou extérieure au système.

Voir aussi : Calibrage.

Anglo-américain : Calibration.

Fausse couleur, n.f.

Domaine : Télédétection électromagnétique.

Définition : Couleur volontairement modifiée pour donner à l'observateur humain une perception colorée d'une * scène, différente de celle qu'il en aurait naturellement.

Note : 1. Ce terme a été introduit par l'invention de l'émulsion couleur incluant une couche sensible au proche infrarouge (appelée I.R.C. ou infrarouge couleur) qui est l'exemple le plus répandu de fausse couleur. Fausse couleur reste généralement employé pour dénommer les émulsions photographiques qui réalisent un déplacement spectral

2. Par extension, on désigne aussi par fausse couleur toute association de bandes spectrales colorées. Anglo-américain : False colo $(u) r$.

Groupe-capteur, n.m.

Domaine : Télédétection électromagnétique.

Définition : Ensemble de *capteurs implantés sur une même *plate-forme pour une mission déterminée et commandés par un dispositif central.

Note : L'ensemble des capteurs à bord d'un satellite constitue un exemple de groupe-capteur.

Anglo-américain : Sensor system.

Image, n.f.

Domaine : Télédétection électromagnétique.

Définition : 1. Représentation plane obtenue à partir d'un enregistrement structuré de données (image potentielle) saisies par *télédétection aérospatiale.

2. Par extension, l'enregistrement qui permet d'obtenir cette représentation.

Note : 1. Un même enregistrement structuré de données peut permettre d'obtenir différentes images réelles. 2. L'image photographique doit être considérée comme un enregistrement analogique particulier. Anglo-américain : Image.

Image composite, n.f.

Voir : Image en couleur composée.

Image composite couleurs, n.f.

Voir : Image en couleur composée.

Image d'écran, n.f.

Domaine : Télédétection.

Définition : *Image temporaire qui ne peut être séparée du *visuel.

Note : 1. Les données affichées sur un *visuel constituent un exemple d'image d'écran.

2. Familièrement, image vidéo.

Anglo-américain : Soft copy.

Image en couleur composée, n.f.

Domaine : Téléđétection électromagnétique.

Définition : *Image en couleur qui résulte de la combinaison d*images monochromes d'une même *scène.

Note : 1. Le terme anglo-américain est apparu pour désigner un traitement en couleur de bandes spectrales du satellite Landsat.

2. Terme à proscrire : image composite couleurs. Le terme image composite a des sens différents selon le domaine d'emploi (cinéma, photographie, télévision en couleur).

Anglo-américain : Composite colo(u)r image.

Image photographique, n.f.

Voir : Image.

Image potentielle, n.f.

Voir : Image.

Image-satellite, n.f.

Voir : Photo-satellite. 
Image-vidéo, n.f.

Voir : Image d'écran.

Imagerie, n.f.

Domaine : Télédétection électromagnétique.

Définition : Ensemble des *images, potentielles ou réelles.

Anglo-américain : Imagery.

Imageur, n.m. ou adj.

Domaine : Télédétection.

Définition : 1 . Générateur $\mathrm{d}$ "images.

2. Qualifie un instrument ou un appareillage qui permet d'obtenir une *image.

Note : On citera, par exemple : radar imageur (en anglo-américain : imaging radar), radiomètre imageur (en anglo-américain : imaging radiometer).

Anglo-américain : 1. Imaging device, imaging system.

2. Imaging.

Infographie, n.f.

Domaine : Télédétection.

Définition : Ensemble des méthodes et des techniques qui permettent de convertir des données en informations graphiques, et inversement, par des moyens informatiques.

Anglo-américain : Computer graphics.

Infrarouge couleur, n.f.

Voir : Fausse couleur.

L.R.C.

Voir : Fausse couleur.

Largeur de bande, n.f.

Voir : Limite de résolution spectrale.

Largeur de couloir, n.f.

Voir : Couloir exploré.

Limite de résolution, n.f.

Voir : Résolution.

Limite de résolution au sol, n.f.

Domaine : Télédétection électromagnétique.

Définition : Plus petite distance au sol de deux points-objets de la *scène apparaissant distincts sur 1**image

Note : La limite de résolution au sol peut être inférieure à celle qu'aurait permis d'obtenir la *limite de résolution spatiale du *capteur compte tenu de l'échelle, grâce à l'emploi de méthodes de traitement appropriées.

Anglo-américain : Ground resolution.

Limite de résolution radiométrique, n.f.

Domaine : Télédétection électromagnétique.

Définition : Plus petite différence d'intensité entre deux niveaux du signal radiométrique détectée par le *capteur utilisé.

Note : Le terme résolution radiométrique est à proscrire en raison de la confusion qu'il introduit entre pouvoir de résolution radiométrique et limite de résolution radiométrique.

Anglo-américain : Radiometric resolution.

Limite de résolution spatiale, n.f.

Domaine : Télédétection électromagnétique.

Synonyme : Limite de séparation spatiale, n.f.

Définition : Demi-période spatiale d'une *scène constituée par une mire dont le contraste est reproduit sur $l^{\prime *}$ image avec une atténuation déterminée.

Note : 1. Le terme résolution spatiale ne doit pas être employé en raison de la confusion qu'il introduit entre pouvoir de résolution spatiale et limite de résolution spatiale.

2. En photographie, on adopte en général la période de la mire au lieu de la demi-période. 
3. Cette définition ne s'applique pas aux *capteurs en hyperfréquence actifs. Anglo-américain : Spatial resolution.

Limite de résolution spectrale, n.f.

Domaine : Télédétection électromagnétique.

Définition : Plus petit écart entre deux longueurs d'onde séparé par le *apteur utilisé.

Note : 1. Le terme résolution spectrale ne doit pas être employé en raison de la confusion qu'il introduit entre pouvoir de résolution spectrale et limite de résolution spectrale.

2. Le terme limite de résolution spectrale ne doit pas être employé pour désigner la largeur de bande du *apteur.

Anglo-américain : Spectral resolution.

Limite de séparation spatiale, n.f.

Voir : Limite de résolution spatiale.

Maille d'échantillonnage, n.f.

Domaine : Télédétection électromagnétique.

Définition : Surface au sol délimitée par le quadrilatère dont les sommets sont les barycentres de quatre *taches élémentaires deux à deux adjacentes.

Anglo-américain : Sampling grid.

Pas d'échantillonnage, n.m.

Domaine : Télédétection électromagnétique.

Définition : Distance qui sépare les barycentres de deux *taches élémentaires consécutives.

Anglo-américain : Sampling interval.

Photo aérienne, n.f.

Voir : Photographie spatiale.

Photo-satellite, n.f.

Domaine : Télédétection électromagnétique.

Définition : 1. Prise de vue photographique effectuée depuis un satellite artificiel.

2. Document obtenu en appliquant cette technique.

Note : On doit distinguer la photo-satellite de l'image-satellite (en anglo-américain : satellite picture) qui est fournie par un *capteur non photographique.

Voir aussi : Photographie spatiale.

Anglo-américain : 1. Satellite(-borne) photography.

2. Satellite(-borne) photograph.

Photographie multibande, n.f.

Domaine : Télédétection électromagnétique.

Définition : 1. Procédé qui permet d'obtenir des images photographiques par l'emploi simultané de plusieurs appareils photographiques chargés soit d'émulsions différentes, soit de la même émulsion avec des filtres différents.

2. Photographies obtenues avec une * chambre multibande.

Anglo-américain : Multispectral photography.

Photographie spatiale, n.f.

Abrév. : Photo spatiale, n.f.

Domaine : Télédétection électromagnétique.

Définition : 1. Prise de vue photographique effectuée hors de l'atmosphère terrestre.

2. Document obtenu en appliquant cette technique.

Note : 1. La *photo-satellite est un cas particulier de la photographie spatiale.

2. L'expression photo aérienne (en anglo-américain : airborne/aerial photograph(y)) ne concerne que les photographies prises à bord d'un *vecteur atmosphérique.

Anglo-américain : 1. Space photography.

2. Space photograph.

Pixel, n.m.

Domaine : Télédétection électromagnétique.

Définition : Plus petite surface homogène constitutive d'une *image enregistrée, définie par les dimensions de la *maille d'échantillonnage. 
1. Dans le cas de la numérisation d'une bande magnétique analogique, par exemple, le pixel est le résultat de l'intégration, selon un pas donné, du signal enregistré sur la bande.

2. Forme abrégée de picture element (en américain, picture est souvent abrégée en pic ou en pix). Anglo-américain : Pixel, picture element (PEL).

Plate-forme, n.f.

Domaine : Télédétection électromagnétique.

Définition : Structure ou support sur lequel peut être installé l'ensemble des *capteurs et de leurs annexes.

Note : 1. La plate-forme peut comprendre des équipements de mesurage ou de commande, de gestion de bord, etc.

2. On distingue généralement deux parties dans un satellite d'observation : la plate-forme et la charge Voir aussi : Vecteur. utile spécifique d'une mission.

Anglo-américain : Platform.

Portée distale, n.f.

Domaine : Télédétection électromagnétique.

Définition : *Distance-temps au sol qui correspond au rayon dont l'angle avec la verticale du *capteur actif est maximal.

Note : 1. Terme propre au *radar à visée latérale.

2. La largeur de couloir (en anglo-américain : swath width) est égale à la différence entre les portées distale et proximale.

Voir aussi : Portée proximale, angle de dépression.

Anglo-américain : Far range.

Portée proximale, n.f.

Domaine : Télédétection électromagnétique.

Définition : "Distance-temps au sol qui correspond au rayon dont l'angle avec la verticale du *capteur actif est minimal.

Note: Terme propre au *radar à visée latérale.

Voir aussi : Portée distale, angle de dépression.

Anglo-américain : Near range.

Pouvoir de résolution, n.m.

Voir : Résolution.

Pouvoir de résolution radiométrique, n.m.

Voir : Limite de résolution radiométrique.

Pouvoir de résolution spatiale, n.m.

Voir : Limite de résolution spatiale.

Pouvoir de résolution spectrale, n.m.

Voir : Limite de résolution spectrale.

Pouvoir séparateur, n.m.

Voir : Résolution.

Programmathèque, n.f.

Domaine : Télédétection.

Définition : 1. Ensemble organisé de programmes de calculateurs accompagnés des documents explicatifs permettant leur emploi par des personnes autres que leurs auteurs.

2. Lieu où sont conservés ces programmes.

Note : Seule, la première définition est normalisée par l'Afnor et l'Iso :

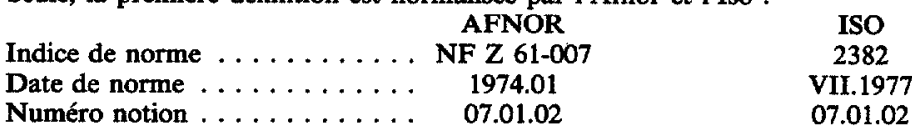

Anglo-américain : Program library.

R.A.A.S., n.m.

Voir : Radar à antenne synthétique. 
Rabattement-radar, n,m.

Voir : Déversement en radar.

Radar à antenne synthétique, n.m.

Abrév. : R.A.A.S., n.m.

Domaine : Télédétection électromagnétique.

Synonyme : Radar à synthèse d'ouverture, n.m.

Définition : *Radar à visée latérale dont le fonctionnement en vol introduit une antenne virtuelle de plus grande dimension que l'antenne réelle par l'utilisation conjuguée de la phase du signal reçu et du déplacement du *vecteur.

Note : 1. Ce mode de fonctionnement permet un accroissement du pouvoir de résolution maximal dans la direction de la trajectoire du *vecteur.

2. Le terme *radar à ouverture synthétique est impropre.

Anglo-américain : Synthetic aperture radar (SAR).

Radar aéroporté à antenne latérale, n.m.

Voir : Radar à visée latérale.

Radar à ouverture synthétique, n.m.

Voir : Radar à antenne synthétique.

Radar à synthèse d'ouverture, n.m.

Voir : Radar à antenne synthétique.

Radar à visée latérale, n.m.

Abrév. : R.V.L., n.m.

Domaine : Télédétection électromagnétique.

Définition : Radar imageur qui explore en vol un couloir décalé latéralement par rapport à la trace du *vecteur.

Notes : 1. Le terme anglo-américain side-looking airborne radar (abrév. SLAR), en français radar aéroporté à visée latérale, désigne l'appareil en mode opératoire aérien.

2. Le terme radar aéroporté à antenne latérale est employé par l'OTAN (réf. 1,1978) comme équivalent de side-looking airborne radar.

Voir aussi : Radar à antenne synthétique.

Anglo-américain : Side-looking radar (SLR).

Radar imageur, n.m.

Voir : Imageur.

Radiomètre à balayage, n.m.

Voir : Scanneur.

Radiomètre imageur, n.m.

Voir : Imageur.

Réalité de terrain, n.f.

Domaine : Télédétection électromagnétique.

Définition : Connaissance de caractéristiques de la *scène étudiée, à partir d'observations et de mesures (enregistrées ou non) réalisées « in situ ".

Notes : 1. Formes à ne pas employer : vérité-terrain ou vérité de terrain en raison des jugements de valeur contenus dans ces expressions.

2. L'ensemble des données disponibles se rapportant à la *scène et obtenu au sol par des moyens appropriés est appelé données de terrain (en anglo-américain : ground data).

3. Faute de pouvoir effectuer, en certaines circonstances, des mesures directement au sol, certains investigateurs utilisent des photographies prises à basse altitude afin d'obtenir sur la *scène des éléments visuels d'interprétation qu'ils désignent par ground truth. Il s'agit en réalité de données auxiliaires de télédétection qui, en toute rigueur, ne peuvent avoir la valeur de données de terrain. De telles données visuelles ne permettent pas, par exemple, d'obtenir toutes les données physicochimiques ou biologiques.

Anglo-américain : Ground truth. 
Résolution, n.f.

Domaine : Télédétection.

Définition : Terme général employé pour évoquer

1. Dans le cas d'un sytème de mesurage, l'aptitude à séparer deux niveaux d'un signal,

2. Dans le cas d'une *image, l'aptitude à rendre distincts deux points voisins.

Notes : 1. La résolution est spécifiée par le pouvoir de résolution (synonyme : pouvoir séparateur, en angloaméricain : resolving power telle que cette notion est retenue en optique (Herschel, Rayleigh, Abbe).

2. On entend par limite de résolution l'inverse de pouvoir de résolution.

3. L'emploi du mot résolution et celui du terme résolution de l'image (traduction littérale du terme anglo-américain : image resolution) sont à déconseiller en raison de la confusion introduite entre la limite de résolution et le pouvoir de résolution.

Anglo-américain : Resolution.

Résolution de l'image, n.f.

Voir : Résolution.

Résolution radiométrique, n.f.

Voir : Limite de résolution radiométrique.

Révolution spatiale, n.f.

Voir : Limite de résolution spatiale.

Résolution spectrale, n.f.

Voir : Limite de résolution spectrale.

Restauration d'image, n.f.

Domaine : Télédétection électromagnétique.

Définition : Ensemble de traitements destinés à rétablir des caractères fondamentaux d'une *image telle que la fournirait un système d'acquisition sans défaut.

Note : Les caractères fondamentaux de l'image les plus couramment rétablis sont les propriétés géométriques et radiométriques.

Anglo-américain : Image restoration.

R.S.O., n.m.

Voir : Radar à synthèse d'ouverture.

R.V.L., n.m.

Voir : Radar à visée latérale.

Scannage, n.m.

Domaine : Télédétection électromagnétique.

Définition : Exploration d'une *scène de façon séquentielle par balayage en vue d'en obtenir une *image.

Anglo-américain : Scanning.

Seanner, v. tr.

Domaine : Télédétection électromagnétique.

Définition : Explorer une *scène de façon séquentielle, par balayage, en vue d'en obtenir une *image.

Note : Ce verbe peut parfois être employé sous la forme intransitive : mettre en ceuvre un *scanneur.

Anglo-américain : To scan.

Scanneur, n.m.

Domaine : Télédétection électromagnétique.

Définition : Radiomètre pourvu d'un dispositif de *scannage qui procède à l'exploration séquentielle d'une * scène, par balayage, afin d'en obtenir une *image.

Note : 1. À titre d'exemple, on citera :

- le scanneur en hyperfréquence (microwave scanner, en anglo-américain);

- le scanneur multibande (multispectral scanner, en anglo-américain).

2. Expression équivalente : radiomètre à balayage.

Anglo-américain : Scanner. 
Scanneur en hyperfréquence, n.m.

Voir : Scanneur.

Scanneur multibande, n.m.

Abrév. : S.M.B., n.m.

Domaine : Télédétection électromagnétique.

Définition : *Scanneur qui procède à une série de données dans plusieurs bandes spectrales simultanément.

Note : Il faut établir une distinction entre le sigle MSS et le sigle M 2 S introduit par la firme Bendix pour désigner un scanneur multibande modulaire (modular multispectral scanner, en anglo-américain).

Anglo-américain : Multispectral scanner (MSS).

Scanneur multibande modulaire, n.m.

Voir : Scanneur multibande.

Scanneuriste, n.m. ou f.

Domaine : Télédétection électromagnétique.

Définition : Personne spécialisée dans la mise en œuvre d'un *scanneur ou d'un appareil similaire.

Scannogramme, n.f.

Domaine : Télédétection électromagnétique.

Définition : *Image d'une *scène obtenue au moyen d'un *scanneur.

Anglo-américain : Scannogram.

Scannographe, $\mathbf{n} . \mathbf{m}$.

Domaine : Télédétection électromagnétique.

Définition : Appareillage qui permet d'obtenir l'enregistrement d'une *scène par *scannage.

Note : Dans le domaine médical (Vocabulaire de la santé et de la médecine, arrêté du 7 décembre 1978), scannographe a été adopté comme substitut au terme anglais scanner pour dénommer l'appareil désigné fréquemment par tomodensitomètre.

Scannographie, n.f.

Domaine : Télédétection électromagnétique.

Définition : Ensemble des connaissances et des procédés techniques qui permettent d'utiliser un *scanneur et les données qu'il saisit.

Note : Dans le domaine médical (Vocabulaire de la santé et de la médecine, arrêté du 7 décembre 1978), scannographie a été adopté pour désigner une méthode de diagnostic utilisant un scannographe.

Voir aussi : Scannographe.

Scannoscope, n.m.

Domaine : Télédétection électromagnétique.

Définition : Appareillage qui permet d'obtenir sur un écran *l'image d'une *scène pendant un *scannage.

Scène, n.f.

Domaine : Télédétection électromagnétique.

Définition : Surface dont le gabarit résulte des propriétés de champ de capteur*.

Anglo-américain : Scene.

Signature spectrale, n.f.

Domaine : Télédétection électromagnétique.

Définition : Ensemble des caractéristiques conditionnant l'interaction du rayonnement électromagnétique avec la matière, nécessaires et suffisantes pour identifier une surface déterminée.

Notes : 1. Parmi ces caractéristiques, les plus employées sont : la réflectance, l'émittance, les effets directionnels, la polarisation.

2. Les propriétés spectrales des cibles présentes dans la *scène n'ont pas l'invariance spationtemporelle des raies spectrales des éléments chimiques simples du fait, en particulier, de l'influence de l'environnement en un lieu de la *scène. De ce fait. la notion de *signature spectrale appliquée à ces cibles constitue une simplification absolue.

Anglo-américain : Spectral signature.

S.M.B., n.m.

Voir : Scanneur multibande. 
Superposition d'images. n.f.

Domaine : Télédétection électromagnétique.

Définition : Procédé qui consiste à faire coïncider géométriquement plusieurs *images, représentant la partie commune d'une *scène, obtenus soit à des dates distinctes, soit au même instant mais dans des bandes spectrales différentes.

Anglo-américain : Images registration.

Tache d'analyse, n.f.

Voir : Tache élémentaire.

Tache élémentaire, n.f.

Abrév. Tachèle, n.f.

Domaine : Télédétection électromagnétique.

Synonyme : Tache d'analyse, n.f.

Définition : Élément d'aire de la *scène résultant de l'intersection du champ de visée instantané ou élémentaire du *apteur avec la surface du terrain.

Notes : 1 . Cette expression ne s'applique pas au capteur photographique.

2. La tache élémentaire dépend, d'une part, de l'angle de la direction principale de visée par rapport au nadir et, d'autre part, de la pente du terrain au lieu visé.

Anglo-américain : Ground patch area.

Tachèle, n.f.

Voir : Tache élémentaire.

T.D.E.M., n.f.

Voir Télédétection.

Téléanalyse, n.f.

Domaine : Télédétection électromagnétique.

Définition : Analyse et traitement, en temps réel ou différé, de données saisies par *télédétection aérospatiale.

Notes : 1. Terme à ne pas employer : téléinterprétation. Ce terme est employé à tort comme forme contractée de télédétection et photo-interprétation.

2. Il n'y a pas d'équivalent anglo-américain connu. On rencontre parfois dans la littérature le terme teleanalysis dont la sémantique ne semble pas suffisamment définie pour être prise en considération et qui ne peut donc être retenu comme équivalent.

Télédétecteur/trice, adj.

Domaine : Télédétecteur électromagnétique.

Définition : Qualifie le professionnel de la *télédétection aérospatiale.

Notes : 1. On citera, par exemple : agronome télédétecteur/-trice ; aménageur télédétecteur/-trice ; géographe télédétecteur/-trice; physicien télédétecteur/-trice; statisticien télédétecteur/-trice.

2. On peut admettre l'emploi substantivé de l'adjectif.

Télédétection, n.f.

Domaine : Télédétection.

Définition : Ensemble des connaissances et techniques utilisées pour déterminer des caractéristiques physiques et biologiques d'objets par des mesures effectuées à distance, sans contact matériel avec ceux-ci.

Note : La télédétection électromagnétique (abrév. T.E.D.M. est un type particulier de *télédétection qui utilise l'interaction du rayonnement électromagnétique avec la matière. Ce terme est à distinguer des techniques aériennes de prospection géophysique (aéromagnétométrie, aérogravimétrie...).

Anglo-américain : Remote sensing.

Télédétection aérienne, n.f.

Voir : Télédétection aérospatiale.

Télédétection aérospatiale, n.f.

Domaine : Télédétection électromagnétique.

Définition : *Télédétection dans laquelle la saisie des données est faite à partir d’appareillages embarqués sur véhicules aériens ou spatiaux.

Notes : 1. Ce terme très utilisé inclut la photographie aérienne.

2. Dans l'état actuel de la pratique, la télédétection aérospatiale ne fait appel qu'au rayonnement électromagnétique. 
3. On traduira airborne remote sensing par télédétection aérienne et spaceborne remote sensing par télédétection spatiale.

Anglo-américain : Airborne and spaceborne remote sensing.

Télédétection électromagnétique, n.f.

Voir : Télédétection.

Télédétection spatiale, n.f.

Voir : Télédétection aérospatiale.

Téléinterprétation, n.f.

Voir : Téléanalyse.

Vecteur, n.f.

Domaine : Télédétection électromagnétique.

Définition : Véhicule aérien ou spatial utilisé pour transporter une *plate-forme et sa charge utile.

Note: Dans le cas des satellites en orbite, la *plate-forme fait parfois office de *vecteur.

Anglo-américain : Selon contexte, platform, vector, vehicle.

Vérité-terrain

Voir : Réalité de terrain.

Vérité de terrain

Voir : Réalité de terrain.

Visualisateur, n.m.

Voir : Visuel.

Visualisation, n.f.

Domaine : Télédétection.

Définition : 1. Procédé qui permet, à partir de données enregistrées, d'obtenir une *épreuve ou une *image d'écran.

2. Cette *épreuve ou cette *image d'écran.

Note : Avant d'aborder l'étape du traitement des images, on effectue souvent une *visualisation rapide à petite échelle (quick look, en anglo-américain) de tout ou partie de l'enregistrement pour en apprécier la qualité.

Voir aussi : Épreuve-minute.

Anglo-américain : Display.

Visualiser, $\mathbf{v}$.

Voir : Afficher.

Visualiseur, n.m.

Voir : Visuel.

Visuel, n.m.

Domaine : Télédétecteur.

Définition : 1 . Dispositif d'affichage ou d'inscription sur un écran ou une console à tube cathodique.

2. Cet écran ou cette console.

Note : Termes à ne pas employer : visualisateur, visualiseur.

Anglo-américain : Display unit.

LISTE 2

Chatoiement, n.m.

Domaine : Télédétection électromagnétique.

Définition : Ensemble de petites taches rapidement fluctuantes qui apparaissent dans la texturation instantanée d'une *image et dû, soit à l'observation à l'aide d'un faisceau cohérent d'une cible présentant des irrégularités à l'échelle de la longueur d'onde, soit à la propagation d'un faisceau cohérent dans une atmosphère caractérisée par des variations aléatoires d'indice ou de réfraction.

Note : Pour des sources ponctuelles (par exemple des étoiles), le terme tavelure est acceptable pour décrire l'aspect des images obtenues en instantané. 
Voir aussi : Effet de mer.

Anglo-américain : Speckle.

Tavelure, n.f.

Voir : Chatoiement. 